\title{
Norois
}

Environnement, aménagement, société

\section{Quel devenir pour les ouvrages hydrauliques installés sur les cours d'eau du bassin de la Maine?}

What is the future of the hydraulic structures along the Maine River basin's watercourse?

Jeannine Corbonnois et Wilfrid Tchékpo

\section{(QpenEdition}

Journals

\section{Édition électronique}

URL : https://journals.openedition.org/norois/4721

DOI : $10.4000 /$ norois. 4721

ISBN : 978-2-7535-2918-2

ISSN : $1760-8546$

Éditeur

Presses universitaires de Rennes

\section{Édition imprimée}

Date de publication : 30 septembre 2013

Pagination : 27-38

ISBN : 978-2-7535-2883-3

ISSN : 0029-182X

\section{Référence électronique}

Jeannine Corbonnois et Wilfrid Tchékpo, «Quel devenir pour les ouvrages hydrauliques installés sur les cours d'eau du bassin de la Maine? ", Norois [En ligne], 228 | 2013, mis en ligne le 30 septembre 2015, consulté le 14 janvier 2022. URL : http://journals.openedition.org/norois/4721 ; DOI : https:// doi.org/10.4000/norois. 4721 


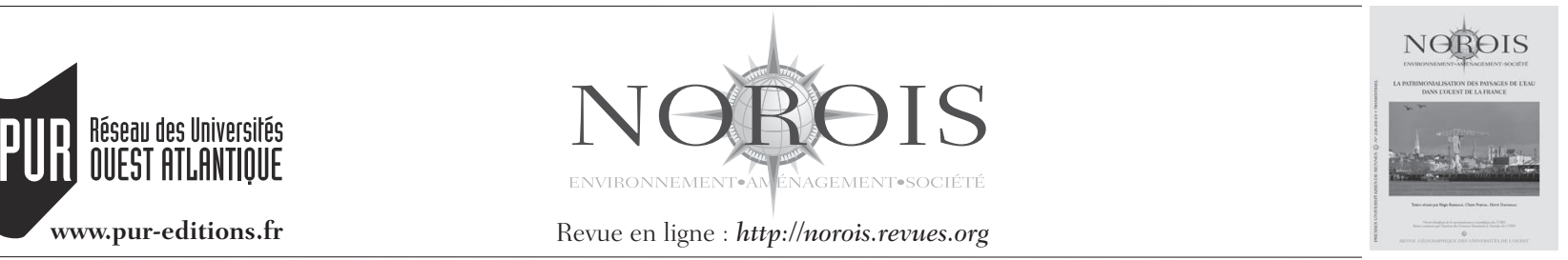

\title{
Quel devenir pour les ouvrages hydrauliques installés sur les cours d'eau du bassin de la Maine?
}

\author{
What Future of the Hydraulic Structures on the Maine River Basin?
}

\author{
Jeannine Corbonnois ${ }^{* a}$, Wilfrid TCHÉKPO ${ }^{b}$
}

\footnotetext{
* Auteur correspondant

a, b PRES LUNAM, université du Maine, UMR CNRS 6590 ESO-Le Mans - 72085 Le Mans cedex 9 (jeannine.

corbonnois@univ-lemans.fr)
}

Résumé : Les ouvrages hydrauliques sont très nombreux le long des cours d'eau du bassin de la Maine. Ils sont plus ou moins bien conservés. La récente loi sur l'eau demande que les continuités écologiques et sédimentaires soient garanties d'ici 2015. Ainsi ces ouvrages sont-ils l'objet de nombreuses discussions et négociations avec leurs propriétaires afin de déterminer leur devenir. Si leur fonction ancienne a depuis longtemps disparu, la fonction actuelle est plus ou moins nettement visible dans le paysage : reconversion en résidence pour les moulins ou en centre d'accueil. La question posée est celle de leur conservation ou de leur aménagement/ arasement. La patrimonialisation de ces installations est une bonne solution pour garantir leur maintien. Mais tous les ouvrages ne pourront pas être préservés. L'article, en présentant d'abord les situations générales dans le bassin de la Maine, discute ensuite de deux cas : l'Erve et le Loir qui présentent des configurations assez représentatives du bassin de la Maine. Les ouvrages participent à la valorisation des paysages de vallées et il faudra faire évoluer encore les mentalités pour faire accepter leur adaptation aux prescriptions de la loi. Parfois, des solutions palliatives sont mises en œuvre afin de coordonner les exigences pour l'environnement et la demande des riverains des cours d'eau et des usagers des vallées.

\begin{abstract}
The hydraulic structures on the Maine River basin, like elsewhere, are quite numerous. They are more or less well preserved. The recent water law requires that ecological and sedimentary continuities are protected by 2015. Thus, these structures focus many discussions and negotiations with their owners in order to determine their futures. If their former function has long since disappeared, their current function is, more or less, clearly visible in the countryside: mills are converted into residences or community centres. The question is whether to preserve them, or to convert/level them. The conversion of these buildings into cultural heritage centres is a good solution to guarantee their continued maintenance, but not all structures can be preserved. This article, after first presenting the general situations in the Maine River basin, discusses the two following cases: the Erve and the Loir rivers which present rather representative configurations from the Maine River basin. The structures contribute to the capitalisation of the valley's landscape. Therefore, in order for the modifications required by the provisions of the law to be accepted, a shift in mentality will need to occur. Sometimes workarounds are implemented to coordinate the requirements of the environment and the demands of the watercourse inhabitants and those who use the valleys.
\end{abstract}

Mots clés : ouvrages hydrauliques - patrimoine fluvial - Maine - paysages fluviaux

Keywords : hydraulic structures - river heritage - Maine - riverscapes. 
Les récents projets de réaménagement des lits fluviaux ont conduit comme dans d'autres pays, à s'interroger sur l'état des ouvrages hydrauliques en vue de leur définir de nouvelles vocations (Walter et Merrits, 2008; Doyle et al., 2003). Ceux-ci constituent par ailleurs un facteur de déclassement des cours d'eau pour l'atteinte du bon état écologique en 2015 (SAGE Loir, 2008). Pour assurer les continuités écologiques et sédimentaires, prescrites par la directive cadre européenne de 2000, ils sont destinés à être arasés, arrangés (abaissement du niveau de l'eau, passe à poissons, etc.) ou aménagés (bras de contournement, intégration à un projet de développement local) (Malavoi, 2003).

En écho aux prescriptions de la DCE, gestionnaires des vallées et des cours d'eau (Agence de l'Eau, Conseil Généraux, CLE des SAGE...) et chercheurs (Germaine, 2011; Barraud, 2011; Lespez et al., 2005 et 2012; REPPAVAL, 2012; Lescure et al., 2011) s'interrogent sur la place des ouvrages hydrauliques dans le paysage, en particulier dans les régions rurales de l'Ouest de la France où se situe le bassin de la Maine (Superficie : $22000 \mathrm{~km}^{2}$ ). Ces ouvrages hydrauliques ont servi à l'alimentation de moulins. Depuis le milieu du $\mathrm{xx}^{\mathrm{e}}$ siècle, la fonction originelle de ces moulins (énergie hydraulique pour produire de la farine, de l'huile, du papier...) a disparu et le plus souvent les bâtiments ont été transformés en habitation (maison individuelle, gîte rural, manoir restauré...). Ainsi la nécessité de rétablir les continuités écologiques et sédimentaires et la disparition de l'intérêt économique des moulins a conduit à reconsidérer l'existence des barrages et à envisager leur arasement.

Cependant cela pose de nombreux problèmes, celui du nouvel état des cours d'eau défaits de leurs aménagements pluriséculaires et celui des paysages ainsi modifiés dans leur configuration générale, (à l'échelle des vallées) et locale (à l'échelle du site) (Barraud, 2011). Une recherche effectuée dans le bassin de la Maine (Corbonnois et al., 2012) a permis de dresser un état des lieux duquel sont extraites nos données. Celles-ci permettent de montrer comment s'élaborent les stratégies destinées à défendre les ouvrages hydrauliques. La mise en patrimoine constitue un instrument de conservation de l'existant, tant des installations principales déjà repérées que des plus petites constituant souvent un patrimoine familial pour leur propriétaire (Germaine,
2010). Mais les situations sont très variées. Les motivations des propriétaires d'ouvrages sont reliées aux contextes économique et social des vallées comme le montrent les exemples des vallées de l'Erve et d'un secteur de Loir (Tchekpo, 2011). Ainsi les actions de restauration écologique et les projets de conservation des héritages souvent difficiles à mettre en cohérence dépendent-ils de commandes externes aux vallées, nationales et européennes, et des commandes internes en lien avec les conditions économiques en particulier le développement touristique des vallées autour d'un patrimoine hydraulique (Barraud, 2009a et b).

\section{Terrain D'ÉTUde ET DONNÉES UTILISÉES}

Les données utilisées sont extraites de la banque de l'ONEMA concernant les ouvrages hydrauliques (Références nationales des obstacles à l'écoulement, état en 2008), des cartes topographiques et des photographies aériennes verticales de l'IGN et obliques (photographies anciennes disponibles aux archives départementales). Elles ont été complétées à partir de relevés de terrain pour les secteurs d'étude. Les travaux de terrain ont permis par ailleurs de collecter d'autres éléments : caractéristiques des vallées et des rivières, sites des ouvrages, définition de leurs fonctions actuelles, identification des projets de reconversion et lien avec le développement local. Vingt-cinq entretiens semi-directifs avec les usagers (propriétaires, association des amis des Moulins, riverains des cours d'eau...) et les gestionnaires (Syndicats de rivière, Direction départementale des territoires, Agence de l'eau Loire Bretagne, Conseil Général de la Sarthe, animateurs des SAGE) ont été réalisés en 2010 et 2011 . Les éléments ainsi collectés servent à mettre en évidence les postures dominantes vis-à-vis de l'application de la DCE et leurs diversités selon la caractéristique des vallées et les fonctions qu'elles remplissent. Ils révèlent aussi l'existence de tensions et de conflits.

Le terrain de l'étude est l'ensemble du bassin de la Maine. On y dénombre plusieurs centaines de barrages et de seuils (figure 1). Leur construction remonte au Moyen Âge et à l'époque moderne (Pichot et Margerie, 2004). Ces installations profitent du caractère peu dynamique des morphologies fluviales en lien avec un drainage de faible énergie 


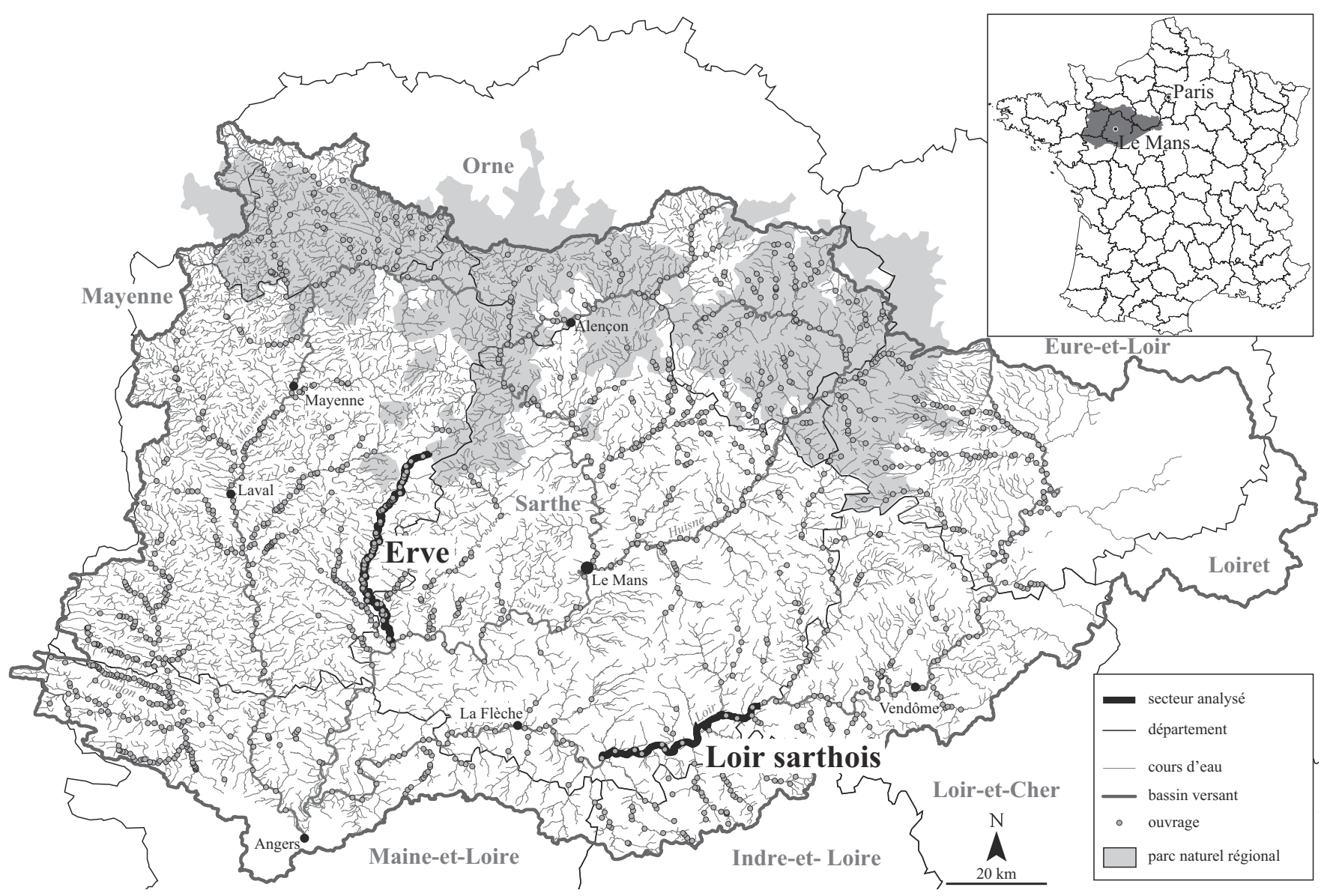

Figure 1 : Les ouvrages hydrauliques du bassin de la Maine (d'après ONEMA et observations de terrain)

The Maine River basin's hydraulic structures (according to ONEMA [The French National Agency for Water and Aquatic Environments] and field observations)

(Corbonnois et al., 2011). Les facteurs décrivant les vallées du bassin de la Maine et les ouvrages hydrauliques sont regroupés dans le tableau 1.

Ces ouvrages continuent d'assurer diverses fonctions selon les lieux. Souvent en milieu rural, où l'activité économique principale est liée à l'agriculture, les ouvrages maintiennent une réserve d'eau. L'activité industrielle de fabrication qui concerne les villes principales et dans une moindre mesure les secteurs ruraux où elle est l'héritière d'une tradition manufacturière issue du XIX ${ }^{\mathrm{e}}$ siècle, sont peu concernés par les installations hydrauliques. Quelques ouvrages alimentent des microcentrales électriques. La présence dans les vallées de nombreux secteurs urbanisés qui fragmentent l'espace rural, introduit dans ces milieux, des enjeux liés à la protection contre les inondations, au maintien de paysages flu- viaux de qualité, au tourisme... (Germaine, 2009; Montembault, 2002; Micoud, 2004).

Ces fonctions expliquent l'attachement d'une grande partie des riverains aux dispositifs actuels. Cet attachement est pris en considération par les instances de gestion. À l'échelle locale (interne aux vallées) des négociations sont conduites par les syndicats de rivières auprès des riverains pour faire émerger un consensus sur le devenir des ouvrages, en particulier en zone rurale où les retenues à l'arrière des barrages servent de réserve d'eau pour l'irrigation en été. À l'échelle plus petite, les acteurs administratifs (ONEMA, Agence de l'Eau, Fédération de pêche...), extérieurs aux vallées, qui prennent en considération les lits fluviaux dans leur globalité, sont chargés de l'application de la loi Cependant tous doivent veiller à la cohérence entre 


\begin{tabular}{|c|c|c|}
\hline $\begin{array}{c}\text { Catégories des } \\
\text { facteurs }\end{array}$ & Facteur & Caractéristiques principales \\
\hline \multirow{4}{*}{$\begin{array}{l}\text { Facteurs d'état } \\
\text { (ou configuration } \\
\text { actuelle) }\end{array}$} & $\begin{array}{l}\text { Conditions natu- } \\
\text { relles }\end{array}$ & $\begin{array}{l}\text { Vallées profondes de } 20 \text { à } 60 \text { mètres, larges de moins de } 2000 \text { mètres. Pentes } \\
\text { longitudinales inférieures à 1/1000. Bonne disponibilité en eau et morphogénèse } \\
\text { fluviale lente. }\end{array}$ \\
\hline & $\begin{array}{l}\text { Aménagements } \\
\text { hydrauliques }\end{array}$ & $\begin{array}{l}\text { Selon la dimension des lits mineurs; chaussée oblique au lit mineur ou seuil } \\
\text { bétonné, chute haute de moins de deux mètres, canal de dérivation long de cin- } \\
\text { quante à quelques centaines de mètres. Moulin à l'architecture variée selon l'âge } \\
\text { et la fonction. }\end{array}$ \\
\hline & $\begin{array}{l}\text { Densité / Propriété } \\
\text { des ouvrages }\end{array}$ & $\begin{array}{l}\text { Très nombreux ouvrages situés sur les plus petits cours d'eau ( } 1 \text { tous les } \\
800 \text { mètres environ), entretenus par les propriétaires regroupés dans les syndicats } \\
\text { de riverains. } \\
\text { Moins d'ouvrages sur les rivières domaniales (destruction d'une quinzaine d'ou- } \\
\text { vrages dès le Xixe siècle pour améliorer la navigation sur la Sarthe aval). }\end{array}$ \\
\hline & $\begin{array}{l}\text { Conditions socio- } \\
\text { économiques }\end{array}$ & $\begin{array}{l}\text { Ouvrages hydrauliques servant à l'élaboration de produits alimentaires et ma- } \\
\text { nufacturés, à la gestion de l'eau pour garantir la ressource (prélèvements pour } \\
\text { l'agriculture et les industries) et à la protection contre les extrêmes hydrologiques. }\end{array}$ \\
\hline \multirow{3}{*}{$\begin{array}{l}\text { Facteurs de la dyna- } \\
\text { mique (modifications } \\
\text { en cours). }\end{array}$} & $\begin{array}{l}\text { Prise en compte de } \\
\text { l'environnement }\end{array}$ & $\begin{array}{l}\text { Impact des ouvrages sur la qualité du milieu naturel (écoulement et qualité de } \\
\text { l'eau, morphologies fluviales, écosystèmes aquatiques) }\end{array}$ \\
\hline & $\begin{array}{l}\text { Nouveaux besoins et } \\
\text { nouvelles exigences } \\
\text { sociétales }\end{array}$ & $\begin{array}{l}\text { Selon les choix des multiples acteurs concernés; demande de paysage de qualité } \\
\text { et pratique de loisirs, modification récente de la fonction des moulins (agrément, } \\
\text { loisirs...), transformation des barrages pour le rétablissement des continuités } \\
\text { écologiques et sédimentaires. }\end{array}$ \\
\hline & $\begin{array}{l}\text { Nouvelles vocations } \\
\text { des ouvrages hydrau- } \\
\text { liques }\end{array}$ & $\begin{array}{l}\text { Selon l'état des installations hydrauliques (abandon/friche, arasement, entretien, } \\
\text { restauration) et les choix effectués (maintien du paysage actuel, de production } \\
\text { d'électricité, patrimoine, gestion de l'eau) }\end{array}$ \\
\hline
\end{tabular}

Tableau 1 : Caractéristiques des vallées et des ouvrages hydrauliques du bassin de la Maine Landscapes characteristics associated with the hydraulic structures of the Maine river basin

les préoccupations pour l'environnement et la posture des riverains des cours d'eau face à la demande de suppression des ouvrages. L'attachement des riverains aux ouvrages est par ailleurs à l'origine de nombreuses protestations coordonnées par les associations de riverains des cours d'eau qui craignent une altération des paysages et une réduction de la qualité des sites, une amplification des extrêmes hydrologiques et un préjudice pour la pêche. Les riverains en faveur de l'arasement des ouvrages ou de leur aménagement manifestent bien moins de véhémence. Il s'agit alors le plus souvent d'ouvrages peu entretenus (barrages de Chammes sur l'Erve amont) ou peu accessibles depuis les routes et chemins (barrage de Varennes sur le Loir), Les arbitrages sont effectués dans le cadre des SAGE par les Commissions Locales de l'Eau (CLE) chargées de définir les objectifs de la gestion des cours d'eau et par les syndicats de rivière; les techniciens de rivières, en contact avec les propriétaires d'ouvrages situés sur les petits cours d'eau jouent un rôle important de médiateur. Les données de la figure 2 sont extraites des entretiens réalisés sur le terrain et de la bibliographie (Barraud, 2009b; Malavoi, 2003).

Ainsi, dans ce bassin de la Maine les enjeux liés aux ouvrages hydrauliques et à leurs fonctions varient selon les lieux et les acteurs : gestion et usages de l'eau, qualités des écosystèmes aquatiques, paysages de vallées, développement local, choix des riverains. Dans le cadre de l'application de la loi imposant le rétablissement des continuités écologiques et sédimentaires, les cas de l'Erve et d'un tronçon du Loir montrent que la nature et l'ordre de prise en considération de ces enjeux sont diversement combinés d'une vallée à l'autre.

\section{LES OUVRAGES HYDRAULIQUES DE L'ERVE ET DU LOIR}

Leurs caractéristiques dépendent de la dimension des vallées et des cours d'eau et de leurs fonctions, en continuité (besoins économiques) ou en rupture (tourisme, loisir, agrément) avec des usages anciens. 


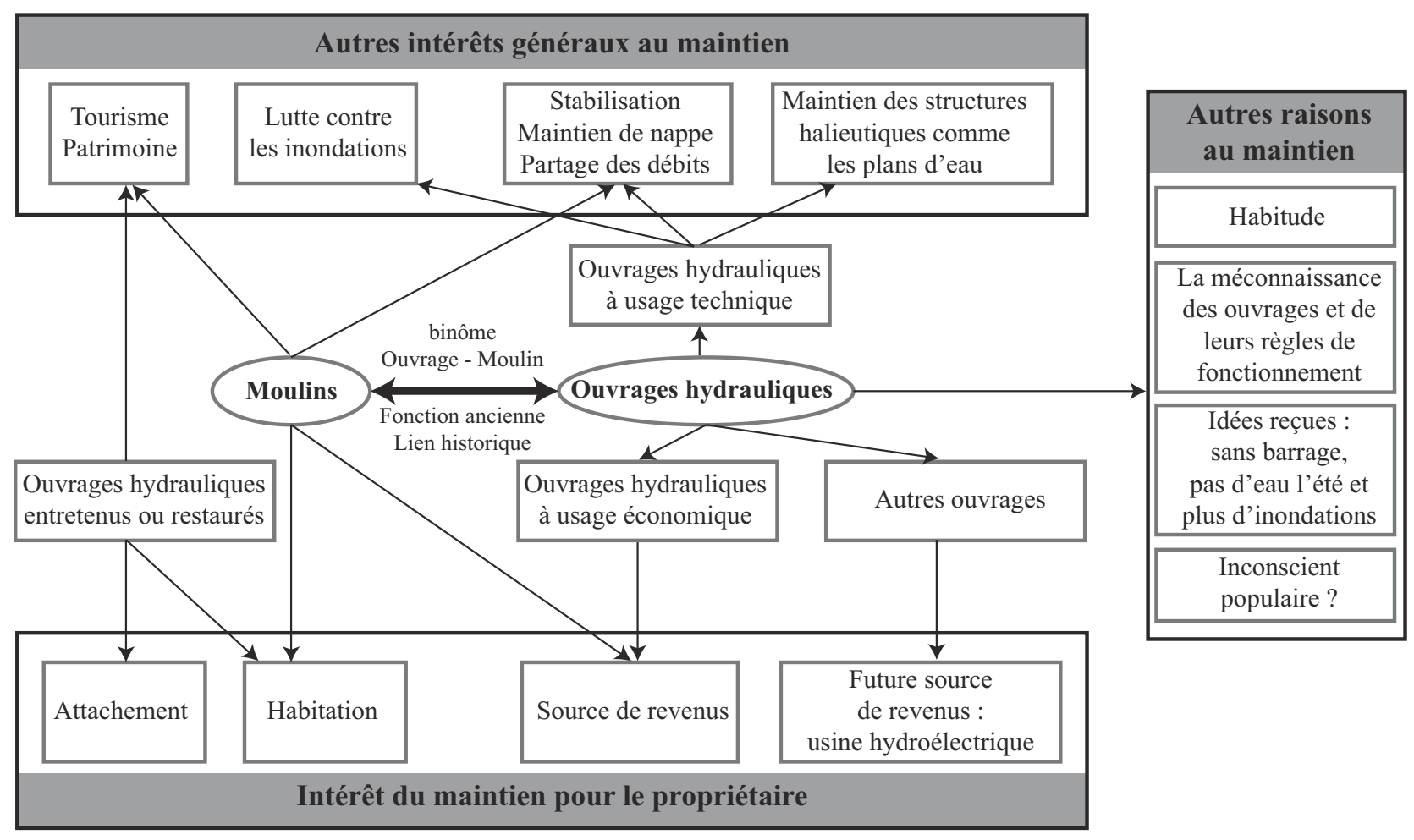

Figure 2 : Fonctions représentées des moulins et ouvrages hydrauliques Representations of the functions of mills and hydraulic structures

\section{Particularité des vallées et catégories d'ouvrages}

L'Erve qui draine un bassin versant de $380 \mathrm{~km}^{2}$ (à la station d'Auvers le Hamon), bénéficie d'une bonne alimentation en eau avec un module de $2,7 \mathrm{~m}^{3} / \mathrm{s}$ $\left(7 \mathrm{l} / \mathrm{s} / \mathrm{km}^{2}\right)$. Elle se jette dans la Sarthe à Sablé sur Sarthe. La rivière est large de moins de $15 \mathrm{~m}$ en général. Elle parcourt une région de plateaux, à la limite du Bassin de Paris et du Massif Armoricain où elle s'encaisse (vallée étroite de moins de 50 à $250 \mathrm{~m}$ ) et profonde de 25 à 30 mètres, avec localement des allures de gorge dans la traversée des calcaires datant de l'ère Primaire (secteur de Saulges). Les activités agricoles dominent largement dans le bassin versant (polyculture/élevage). Les aménagements hydrauliques -48 au total - (figures 3 et 4), remis en état pour les $2 / 3$ au cours des années 1970/1980 servent au maintien des niveaux d'eau. Il s'agit le plus souvent d'un seuil perpendiculaire aux berges, équipé d'une vanne mobile. Mais l'Erve parcourt aussi une région où le tourisme rural constitue une ressource économique notoire. Cela est en lien avec des paysages naturels variés, reliefs originaux de Sainte-Suzanne et de Saulges, zones naturelles classées. Ces caractéristiques ont valu à cinq de ces ouvrages d'être classés comme patrimoine historique et culturel. Les autres ouvrages sont majoritairement des moulins convertis en résidences dont certains propriétaires réclament également une mise en patrimoine (figures 5 et 6) (Tchékpo, 2011).

Le secteur du Loir pris en considération est situé dans la moitié aval de la vallée, entre Ruillé sur Loir et Bazouge. Le cours d'eau qui draine un bassin versant de $7920 \mathrm{~km}^{2}$ écoule un débit de $31,6 \mathrm{~m}^{3} / \mathrm{s}$ soit $4 \mathrm{l} / \mathrm{s} / \mathrm{km}^{2}$ (station de Durtal). Il parcourt des bas plateaux établis dans les grès, sables et calcaires (d'âge fini Secondaire et Tertiaire) de l'ouest du Bassin parisien. La vallée est profonde d'une trentaine de mètre et sa largeur est de l'ordre de $2000 \mathrm{~m}$. Les activités économiques y sont plus variées (agriculture, industries, activités tertiaires...) que dans la vallée de l'Erve. Les villes et bourgs, plus nombreux, lui valent une plus grande densité de population et des usagers aux intérêts variés pour les ouvrages hydrauliques. Le tronçon pris en considération est géré par le Conseil Général de la Sarthe (rivière domaniale déclassée). Les installa- 


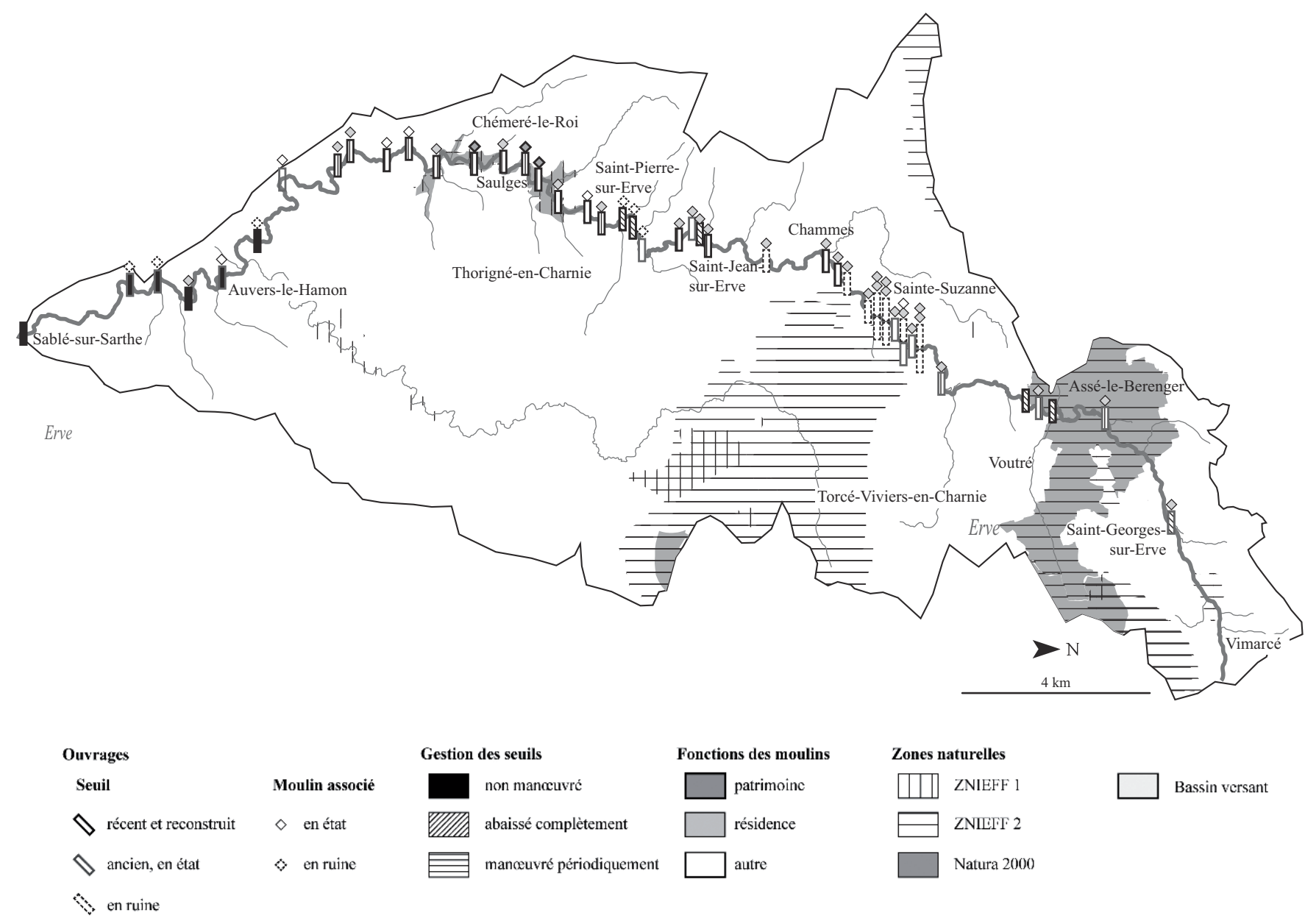

Figure 3 : Caractéristiques des ouvrages hydrauliques du bassin de l'Erve Functions of the Erve's hydraulic structures

tions hydrauliques nombreuses (26 au total) sont, en raison de leur grande dimension, bien visibles dans le paysage. En effet, il s'agit de longues chaussées barrant obliquement la rivière dont environ la moitié atteint plus de $200 \mathrm{~m}$ de longueur. Par ailleurs, les moulins liés à ces ouvrages sont de taille imposante, plusieurs d'entre eux ont été agrandis au XIX ${ }^{\mathrm{e}}$ siècle pour la production de farine et de papier et certains sont associés à un château.

Le tableau 2 comprend les principales catégories d'ouvrages qui ont été déterminées à partir de données collectées. Elles prennent en considération le barrage et le moulin qui y est associé. Ceux-ci sont repérés sur les figures 3 et 4 .

\section{Fonctions actuelles et processus de patrimonialisation des ouvrages hydrauliques}

Les fonctions actuelles des ouvrages hydrauliques sont déterminées de diverses manières par l'environnement naturel, culturel et historique des vallées. Le statut des rivières, les choix économiques du développement local et les modalités de la gestion des infrastructures hydrauliques jouent également un rôle important. Quelques exemples, qui tiennent compte des caractéristiques des vallées, montrent comment l'environnement des moulins et barrages sert à justifier qu'ils soient conservés pour leur intérêt patrimonial.

Sur l'Erve, ce sont les particuliers qui défendent les ouvrages hydrauliques (figure 3). Le moulin de Thévalles est un établissement privé associé à un 


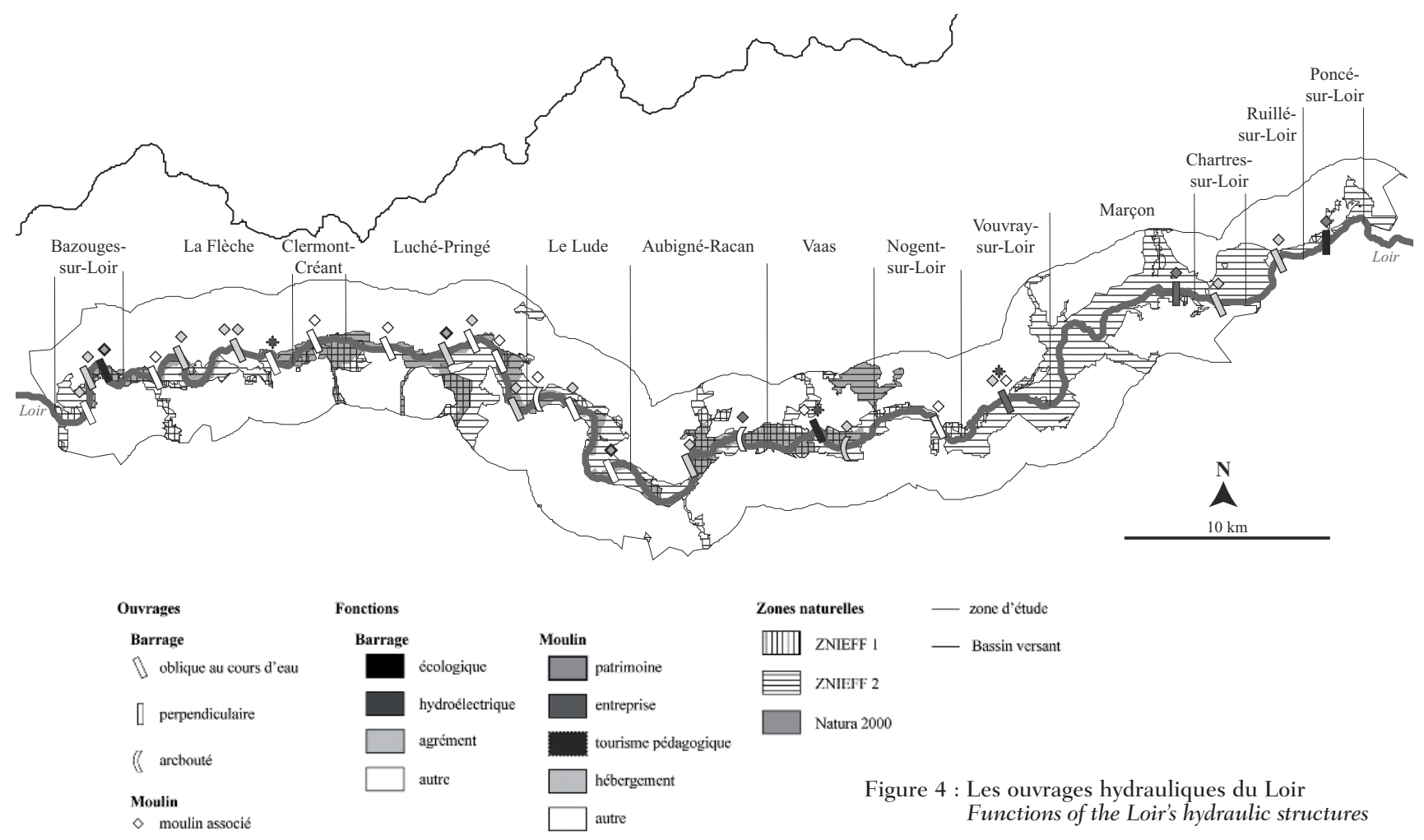

\begin{tabular}{|c|c|}
\hline Nature du propriétaire & Caractéristiques de la gestion \\
\hline Rachat par un particulier & $\begin{array}{l}\text { L’acquéreur s'est assuré de la rentabilité de son investissement en regard au nouvel usage } \\
\text { qu'il entend faire du site. Plusieurs moulins ont été transformés en hôtel-restaurants (la } \\
\text { Courbe, le moulin des } 4 \text { saisons de la Flèche, le moulin de Pousset, de Martinet etc), ou en } \\
\text { gîtes d'accueil à l'instar de Chérré }\end{array}$ \\
\hline $\begin{array}{l}\text { Rachat par la commune (moulin de Bruère, } \\
\text { papeterie de Paillard.). Il s'agit ici d'un achat } \\
\text { de transition car en réalité les ouvrages ne } \\
\text { sont pas sous la tutelle directe des com- } \\
\text { munes. Ils sont concédés à des privés ou des }\end{array}$ & $\begin{array}{l}\text { La commune de la Flèche, par exemple, en rachetant le moulin de la Bruère en } 1994 \text {, } \\
\text { affichait une volonté claire de voir se pérenniser la production de pains de glace. Dans le } \\
\text { cas du moulin de Paillard, la cession a d'abord lieu entre particuliers puis la commune ra- } \\
\text { chète le site aux fins de le sauvegarder comme patrimoine local. Actuellement, de nouveaux } \\
\text { exploitants s'occupent de son animation par l'entremise d'un contrat avec la commune de } \\
\text { Poncé-sur-Loir (centre artisanal) }\end{array}$ \\
\hline $\begin{array}{l}\text { Racha } \\
\text { l'admi }\end{array}$ & $\begin{array}{l}\text { Exemple du barrage de Ruillé : il a été cédé à un particulier, propriétaire de parcelles agri- } \\
\text { coles situées tout autour de l'ouvrage. Mais le niveau de l'eau n’a jamais pu être réglé afin } \\
\text { de permettre l'irrigation en raison du mauvais état de l'ouvrage mais surtout de l'absence } \\
\text { du droit d'eau qui autoriserait des interventions sur la chute d'eau. De nombreux recours } \\
\text { ont été déposés, sans succès. entre } 1982 \text { jusqu'en } 2000 \text {; avec l'avènement des nouvelles } \\
\text { règlementations sur la gestion des cours d'eau communautaires une fin de non recevoir de } \\
\text { ses doléances a été notifiée au propriétaire. La proposition qui lui a été faite in fine est de } \\
\text { laisser l'ouvrage en l'état. }\end{array}$ \\
\hline $\begin{array}{l}\text { Valorisation des } \\
\text { parents d'ancien }\end{array}$ & $\begin{array}{l}\text { C'est le cas du moulin de Rotrou, du moulin du Bazouges, de la Noria de Malidor (quoiqu’un } \\
\text { vieux projet de mise en valeur de ce site semble avoir existé), du moulin Neuf, etc. }\end{array}$ \\
\hline $\begin{array}{l}\text { Ouvrages n'ayant fait l'objet d'aucune atten- } \\
\text { tion, installation délabrée. }\end{array}$ & $\begin{array}{l}\text { C'est le cas du moulin de Saint Jacques, du moulin de la Courante, du moulin des Îles et } \\
\text { même du grand moulin de Vaas dont les bâtiments n'ont subi aucune transformation remar- } \\
\text { quable depuis la fin de leurs activités. }\end{array}$ \\
\hline
\end{tabular}

Tableau 2 : Catégories d'ouvrages hydrauliques selon leurs fonctions actuelles

Classification of hydraulic structures according to their current functions 


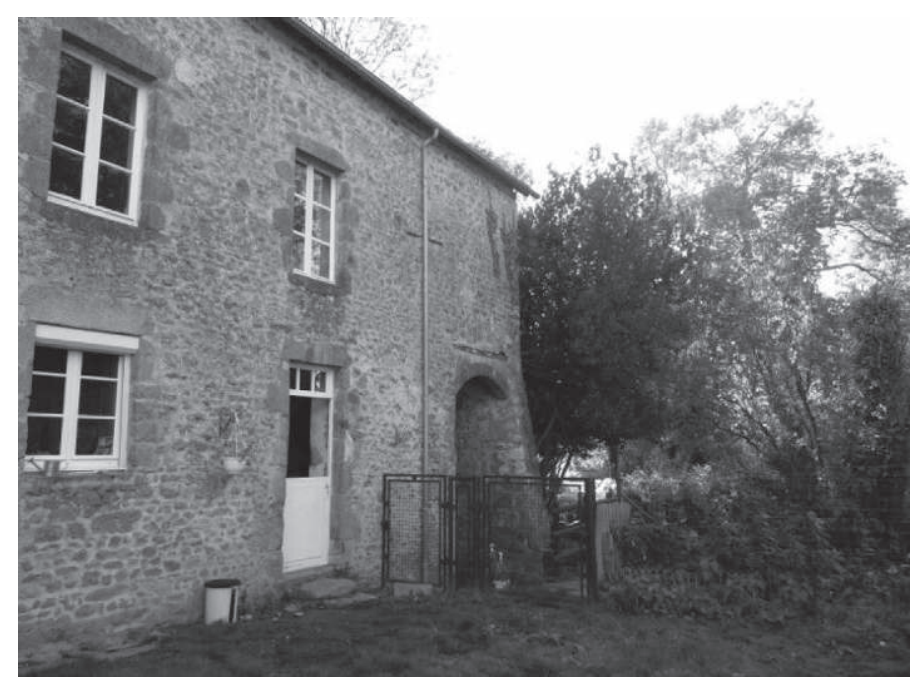

Figure 5 : Moulin d'Assé, devenue résidence permanente (Erve) (Source : Tchékpo W., 2010) 2010)

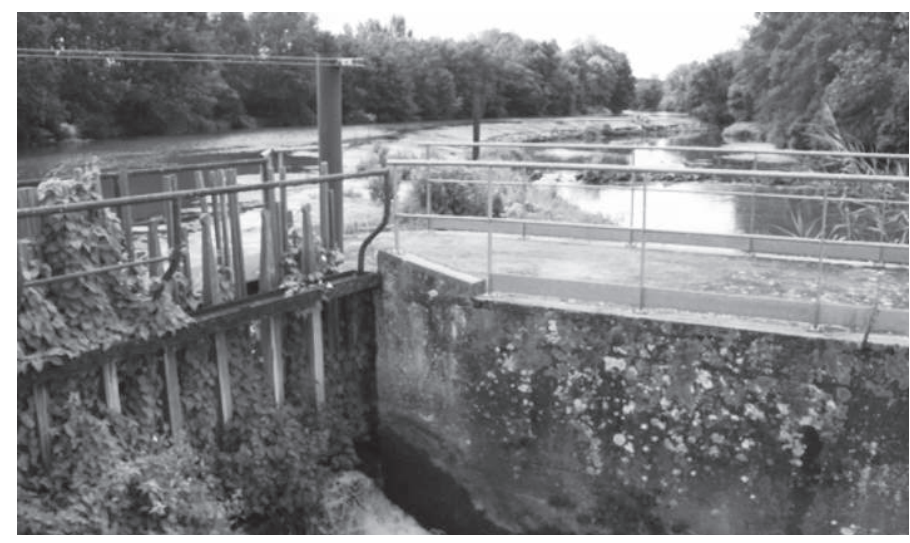

Figure 6 : Ouvrage hydraulique de Varennes (Loir) (Source : J. Corbonnois 2011)

Varennes hydraulic structure (Loir) (Shot: J. Corbonnois 2011)

château. Le site est intégré depuis juillet 1977 dans le périmètre d'une ZNIEFF, de type 1 avec la commune de Saulges et de type 2 avec les communes de Saulges, de Ballée, de Saint-Pierre-sur-Erve, et de Throignée-en-Charnie. Le site du moulin intègre aussi le périmètre du premier site Natura 2000 de la Région Pays-de-la-Loire, accordé à la vallée de l'Erve en décembre 2003. Depuis la fin de son activité (1958) de production de farine et de micro hydroélectricité le moulin (première mention en 1260), a été progressivement restauré. En 1995, le site (moulin et château) est ouvert au public et il accueille une exposition permanente de maquettes de moulins. Régulièrement, la roue est mise en marche pour le plaisir des nostalgiques et pour éveiller la conscience des nouvelles générations sur la richesse historique et patrimoniale des moulins. Le moulin du Pont du Gué est site classé à ressources naturelles et historiques. Il est en plein cœur des Grottes de Saulges (vestiges préhistoriques) qui s'étendent sur près de $2 \mathrm{~km}$ (du moulin de la Roche Brault jusqu'au pont de Gué). Il est classé à l'inventaire Natura 2000 (1977). Le monument lui-même est inscrit au patrimoine historique en 2003. Le moulin a cessé son activité dans les années 1920. Un lavoir communal situé sur le barrage en amont du moulin était fréquenté jusqu'en 1864. Actuellement, un restaurant, des sentiers de randonnées et un mur d'escalade entretiennent une grande fréquentation du site. C'est le seul lieu dans l'Ouest de la France où une vallée en gorge est taillée dans les calcaires du Primaire karstifiés ce qui pourrait lui valoir de ce fait, une identification comme "géomorphosite " (Rivas et al, 1997, cité par Reynard, 2005) (figure 7).

D'autres établissements constituent un patrimoine plus ordinaire. « Les moulins deviennent l'archétype des paysages bucoliques et champêtres. Les rivières et leurs aménagements sont partie prenante d'un nouveau cadre de vie et engendrent des activités sportives, ludiques et contemplatives » (Barraud, 2009b). Le moulin est un patrimoine hérité à entretenir et à transmettre afin que la mémoire familiale et historique se pérennise. Certains anciens moulins ont été transformés en gîtes ou en résidences secondaires ou mis en location (exemple du moulin de Gratte Sac à Voutré) ; l'histoire du moulin tient une place de choix dans la promotion de ces résidences mais aussi la promotion de la pêche en eau calme (bief des moulins).

Sur le Loir (figure 4), où la navigation fluviale est abandonnée dès le XIX ${ }^{\mathrm{e}}$ siècle, la situation des ouvrages est le résultat de choix successifs, effectués par l'état puis par des propriétaires qui ont progressivement racheté les moulins (tableau 3).

\section{Discussion}

Les exemples de l'Erve et du Loir montrent quelles sont les stratégies mises en œuvre par les propriétaires des installations hydrauliques pour les conserver. L'argument principal est que ces installations contribuent au développement local des vallées en dynamisant le tourisme surtout dans les 
Figure 7 : Géomorphosite, modèle des cinq valeurs autour de formes de relief. Une adaptation du modèle de Reynard (2005). Application aux grottes de Saulges Geomorphosite, model of the five values around landforms. An adaptation of the Reynard's model (2005) applied to the Saulges caves

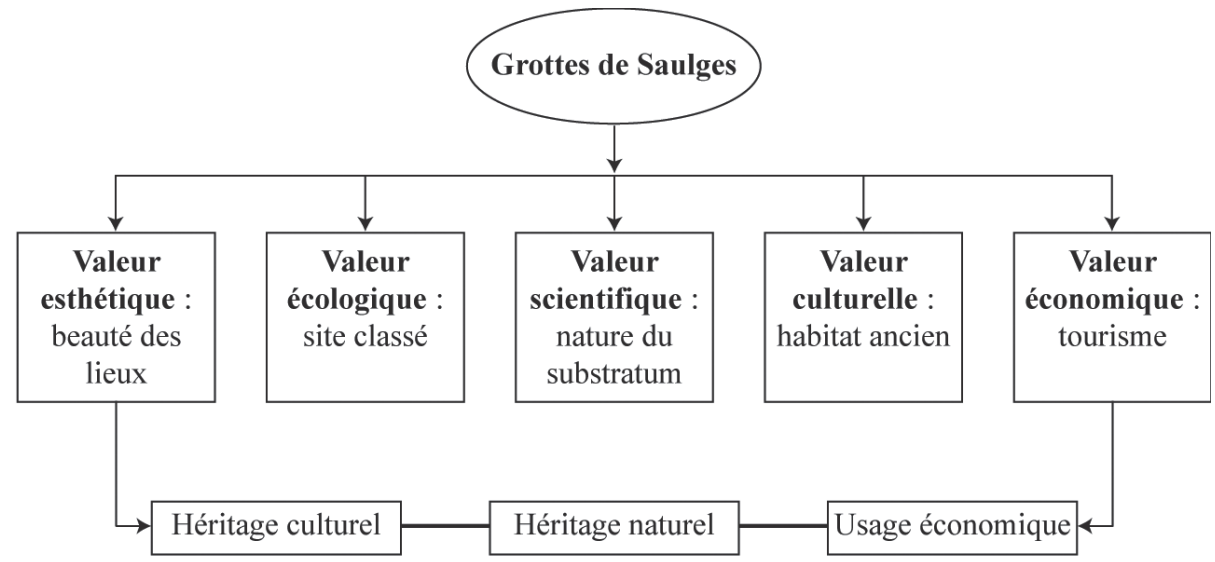

\begin{tabular}{|c|c|c|c|}
\hline Catégorie & Définition & Fonction & Exemples \\
\hline $\begin{array}{l}\text { Ouvrages «patri- } \\
\text { moine » }\end{array}$ & $\begin{array}{l}\text { Poids du passé historique des lieux, } \\
\text { attraction actuelle auprès du public } \\
\text { français et étranger, intérêt des sites } \\
\text { naturels d'implantation et exploi- } \\
\text { tation à des fins de mise en valeur } \\
\text { touristique. }\end{array}$ & $\begin{array}{l}\text { Participation à la fonction récréa- } \\
\text { tive des vallées et contribution au } \\
\text { développement touristique culturel } \\
\text { et pédagogique }\end{array}$ & $\begin{array}{l}\text { Moulin, du château de Thévalles et } \\
\text { vallée de Saulges sur l'Erve, } \\
\text { Moulin de Rotrou, Moulin de Ba- } \\
\text { zouge sur le Loir }\end{array}$ \\
\hline $\begin{array}{l}\text { Propriété privée } \\
\text { restaurée pour } \\
\text { l'habitation }\end{array}$ & $\begin{array}{l}\text { Moulin refait systématiquement } \\
\text { occupé par les descendants d'an- } \\
\text { ciens propriétaires ou de nouveaux } \\
\text { occupants ayant acheté le site. } \\
\text { Certaines constructions ont gardé } \\
\text { leur configuration ancienne }\end{array}$ & $\begin{array}{l}\text { Moulins restaurés, équipés d'une } \\
\text { roue alimentée à partir d'un seuil } \\
\text { dont l'état est variable. Fonction } \\
\text { d'agrément }\end{array}$ & Moulin de Feuillaume sur l'Erve \\
\hline $\begin{array}{l}\text { Propriété privée } \\
\text { ouverte au public }\end{array}$ & $\begin{array}{l}\text { Bâtiment d'ancien moulin plus ou } \\
\text { moins restauré, servant à des asso- } \\
\text { ciations, des hébergements collec- } \\
\text { tifs... (gîte rural, hôtel). }\end{array}$ & $\begin{array}{l}\text { Participation à l'activité économique } \\
\text { et culturelle, seuil entretenu pour la } \\
\text { gestion de l'eau (agriculture) et les } \\
\text { loisirs (pêche, canoë). Ponctuelle- } \\
\text { ment production d'électricité }\end{array}$ & $\begin{array}{l}\text { Moulin de la Roch Brault sur l'Erve, } \\
\text { Moulin de Chérré, de Courbe de } \\
\text { Martinet à Coëmont sur le Loir }\end{array}$ \\
\hline $\begin{array}{l}\text { Barrage sans mou- } \\
\text { lin remis en état ou } \\
\text { construit au cours } \\
\text { des années } 70-80\end{array}$ & $\begin{array}{l}\text { Barrage équipé de vannes mobiles } \\
\text { actionnées par le syndicat de rivière }\end{array}$ & $\begin{array}{l}\text { Maintien des niveaux d'eau et régu- } \\
\text { lation des écoulements }\end{array}$ & $\begin{array}{l}\text { Tous les barrages de l'Erve et } \\
\text { d'ailleurs sur toutes les petites } \\
\text { rivières (absents sur le Loir) }\end{array}$ \\
\hline $\begin{array}{l}\text { Ouvrage sans fonc- } \\
\text { tion spécifique }\end{array}$ & $\begin{array}{l}\text { Ancien moulin dont l'usage actuel } \\
\text { n'a plus de lien avec les fonctions } \\
\text { anciennes. }\end{array}$ & $\begin{array}{l}\text { Dispositif conservé mais déconnecté } \\
\text { des établissements industriels anté- } \\
\text { rieurement alimentés }\end{array}$ & Moulin de Varennes sur le Loir \\
\hline $\begin{array}{l}\text { Site complètement } \\
\text { abandonné }\end{array}$ & $\begin{array}{l}\text { Maison d'habitation construite à la } \\
\text { place de l'ancien moulin, absence de } \\
\text { traces de l'activité ancienne }\end{array}$ & $\begin{array}{l}\text { Vestiges de barrage et ruines (an- } \\
\text { cienne chaussée végétalisée). }\end{array}$ & $\begin{array}{l}\text { Moulin de Gô sur l'Erve, Vieux mou- } \\
\text { lin à Marçon sur le Loir }\end{array}$ \\
\hline
\end{tabular}

Tableau 3 : Types et objectifs de la conservation des ouvrages hydrauliques du Loir Conservation types and objectives for the hydraulic structures of the Loir

régions rurales. En effet, elles entretiennent et parfois amplifient l'offre d'activités culturelles, de loisirs et d'accueil. De plus, leur intérêt s'additionne à ceux des autres composantes remarquées des espaces ruraux (bocage, habitat, architecture...).
Dans la vallée de l'Erve, trois communes sont distinguées «Petites Cités de Caractères » (SaintPierre-sur-Erve, Sainte-Suzanne et Saulges). Ce sont là des atouts supplémentaires. Des événements sont organisés pour célébrer les moulins : journée Nationale des Moulins, journée des Peintres de 
Saulges ou du Patrimoine de l'Europe, route des moulins à Sainte-Suzanne.

Dans celle du Loir, la situation socio-économique, plus favorable, tire profit de l'ambiance paysagère qui est déjà celle de l'Anjou et de la vallée de la Loire située à quelques dizaines de kilomètres au sud de ce secteur (Gatien et al., 2009; Hydroconcept, 2003). Pour le département de la Sarthe, la vallée du Loir est un milieu d'attraction touristique exceptionnel. Les nombreux intérêts sont liés aux paysages (vallée ample et occupation diversifiée du sol comprenant en particulier des vergers et de la vigne), aux milieux naturels variés et à leur richesse floristique et faunistique (figure 4), aux constructions présentant souvent une architecture harmonieuse, (habitat en tuffeau, manoirs, moulins...). De nombreuses localités y sont distinguées «Pays d'art et d'histoire ». « De La Chartre sur le Loir à La Flèche, la vallée du Loir sarthoise est le point de convergence architectural de l'Anjou, du Baugeois et de la Touraine. Elle constitue de ce fait l'une des unités architecturales des plus typées du département de la Sarthe et forge son identité autour des vergers et de la vigne qui modèlent encore les paysages de coteaux. » (Conseil Général de la Sarthe, 2010). De nombreuses localités y sont distinguées «Pays d'art et d'histoire ».

Dans ces deux vallées, la demande pour le maintien au moins de certains ouvrages reste très forte. Mais l'Erve a été classée sur la liste 2 ce qui signifie que les continuités sédimentaires et écologiques doivent être assurées d'ici 5 ans. La mission des syndicats de rivières est dorénavant de faire aux propriétaires d'ouvrages, des propositions chiffrées et cohérentes permettant d'atteindre les objectifs. Cependant ces derniers voient là un moyen pour les conduire à accepter l'arasement de nombreux ouvrages ou bien leur gestion selon les préconisations de l'administration.

On peut noter également que les intérêts pour l'environnement influencent diversement les politiques tant au plan local que départemental. Parfois, la préservation d'un écosystème remarquable, nécessite d'envisager que des ouvrages hydrauliques soient conservés; ils maintiennent un niveau élevé et peu variable d'eau dans les rivières et dans les nappes souterraines et contribuent à stabiliser ou à réguler le milieu de vie des espèces fauniques et floristiques abritées par les zones naturelles. Aussi, le barrage de Vaas sur le Loir est-il en réhabilitation (aménagement pour que les continuités écologiques et sédimentaires soient assurées) pour garantir la préservation de la zone humide de la Prée d'Amont d'une superficie de plusieurs dizaines d'hectares. D'autres ouvrages pourraient être traités de manière similaire puisque le secteur du Loir dans la traversée du département de la Sarthe, est couvert de zones naturelles et également de monuments historiques (châteaux et ouvrages hydrauliques associés). L'atlas des paysages du département de la Sarthe (2010) propose de préserver ces milieux de la vallée du Loir au regard de leur sensibilité et de leur originalité, que la pérennité de certains ouvrages soit garantie dans la mesure où leur destruction est perçue comme un danger pouvant entraîner la chute du niveau du Loir.

Ainsi, notre analyse souligne-t-elle les difficultés de la mise en ouvre de la DCE et donc de l'atteinte du bon état écologique des rivières. L'Erve, par la densité des ouvrages (figure 1), est assez représentative des situations qui caractérisent les affluents des rivières principales, non domaniales, inscrites en milieu rural. Dans le bassin de la Maine, des situations similaires ont été observées et analysées sur la Vègre ou encore l'Oudon (affluent de la Mayenne) ou la Braye (affluent du Loir). Souvent les riverains des cours d'eau qui sont conscients des problèmes posés par la morphologie des lits fluviaux et la continuité piscicole, protestent avant tout contre les modalités de la mise en œuvre de la DCE. Ils réclament une plus grande concertation. Ainsi depuis deux ou trois ans, des négociations sont faites individuellement avec les propriétaires d'ouvrages. Sur les rivières de gabarit supérieur, les demandes de maintien d'ouvrages sont diversement argumentées. Si sur le Loir, la conservation d'un paysage de qualité est privilégiée, sur la Sarthe en amont du Mans, ce sont les attentions reliées à la gestion des inondations qui occupent la plus grande place (Corbonnois et al., 2012). Le Conseil général de la Sarthe a désigné les ouvrages en friche qui devront être détruits.

Enfin, il faut souligner qu'on ne connait encore que très incomplètement le fonctionnement hydrogéomorphologique des lits fluviaux qui se sont ajustés depuis plusieurs siècles aux barrages et ainsi à un transfert perturbé de la charge sédimentaire; quelle sera la quantité et la qualité des alluvions 
remobilisées du fait de l'arasement des barrages, compte tenu de la faiblesse de l'énergie des cours d'eau, en combien de temps un hydrosystème sera-til renaturé? Finalement la relative lenteur à laquelle les arasements sont réalisés permet d'étaler leurs effets sur les milieux naturels (Lescure et al., 2011; Corbonnois et al., 2011).

\section{Conclusion}

Les installations hydrauliques assurent de nombreuses fonctions, plus ou moins visibles dans le paysage et souvent éloignées des fonctions d'origine. Ainsi les ouvrages du bassin de la Maine sont-ils à l'image de ceux d'autres secteurs de l'ouest de la France et probablement d'autres régions (Lespez, 2012). Les conditions naturelles des vallées et des cours d'eau agissent sur la dimension des ouvrages (longueur des chaussées, hauteur de chute) mais interviennent peu sur leur nombre, qui était commandé par des facteurs anthropiques. L'étude montre comment se sont mis en place les processus de défense des ouvrages hydrauliques, révélant une grande sensibilité sociale. Celle-ci a émergé dans différents secteurs de vallée, plus ou moins dynamique au plan économique ou intéressant au plan écologique.

Les ouvrages dont l'intérêt patrimonial est avéré seront probablement conservés; leurs fonctions répondent aux attentes actuelles de loisir et de culture et certains sont intégrés à un espace classé ou qui pourrait le devenir. Ils seront aménagés pour répondre aux prescriptions de la DCE. Et les autres installations souvent en friche doivent-elles être considérées comme menacées? Les arasements d'ouvrages déjà réalisés sur certains cours d'eau montrent qu'après avoir été réticents, riverains et propriétaires perçoivent favorablement le rétablissement des continuités sédimentaires et écologiques, surtout lorsque les barrages ne sont plus associés à un moulin (nombreux cas sur l'Erve). Là où l'arasement n'est pas possible (moulin et roue restaurés), des aménagements spécifiques, en accord avec les propriétaires sont négociés (bras de contournement du barrage, abaissement de la vanne, passe à poissons...). Le coût des travaux de mise en conformité avec la loi est subventionné par l'état (Agences de l'Eau). Les associations de protection des moulins posent également la question de la production d'hy- droélectricité en particulier sur les grands cours d'eau.

La marge de manœuvre des services en charge de la gestion des cours d'eau est étroite entre la promotion du développement touristique et économique fondée sur la valorisation du paysage, comprenant en particulier les ouvrages hydrauliques, et les nécessités de rétablir les continuités écologiques et sédimentaires sur les cours d'eau. La patrimonialisation des ouvrages a finalement un coût élevé (Cottet et al.; 2010 ; Fernandez et al., 2011).

\section{Remerciements}

Les auteurs tiennent à remercier les deux relecteurs anonymes pour leurs remarques constructives qui ont permis d'améliorer nos analyses et démonstration ainsi que le Comité de rédaction de la revue.

\section{Bibliographie}

Alhaskeer Z., 2012. Analyse cartographique de la structure des paysages de vallées. - évaluation de la dynamique des paysages de vallées du bassin versant de la Maine à partir de la télédétection et de SIG. Thèse de doctorat. Université du Maine. 520 p. + annexes.

Collectif, Bulletins de l'Association des amis des moulins de la Sarthe, Revues parues entre 1990 et 2008.

Collectif, 1991. Le Loir. Archives et documents sarthois. Dossier n 20. Ed. Ardos, Conseil Général Sarthe, 103 p.

Richard V., 2010. Vallée du Loir, destination vignoble. $1^{\text {res }}$ rencontres interrégionales « Enologie en Val de Loire ». Association de développement de la Vallée de la Loire. Fontevrault, 11 p.

BARRAud R., 2009a. La rivière aménagée et le moulin à eau. Un héritage en déshérence? Trajectoires, modèles et projets de paysages. Exemples des vallées sud-armoricaines, Bulletin de l'Association de géographes Français, p. 32-45.

Barraud R., 2009b. Approche géographique de l'intentionnalité paysagère dans les vallées de l'Ouest de la France (de la fin du XVIII ${ }^{\mathrm{e}}$ siècle à aujourd'hui), Projets de Paysage, $\mathrm{n}^{\circ} 1$, [http://www.projetsdepaysage.fr/fr/enquetes_et_debats].

Barraud R., 2011. Rivières du futur/Wild Rivers, VertigO - la revue électronique en sciences de l'environnement. Hors-série 10, [http://vertigo.revues.org/].

Conseil général de la Sarthe, 2010. Atlas des paysages de la Sarthe, 141 p. [www.pays-de-la-loire.developpement-durable. gouv.fr].

Corbonnois J., Rollet A.-J., Viel V., Honoré A., 2012. The DCE, hydraulic structures and bed erosion in the Huisne River (France), dans Arnaud-Fassetta G., Reynard (éd.). Continental hydrosystems and territory and the different laws 
and practices of water mangement in Europe/Hydrosystèmes continentaux et territoires européens confrontés aux différentes lois sur l'eau, Pfeil, Munich, p. 137-148.

Corbonnois J., Tchekpo W., Cheynier L., Mertelmeyer L., Alhasker Z., Gatien A., 2012. Moulins et barrages $d u$ basin de la Maine. Volet 2. dans Patrimoine et Trajectoires paysagères des vallées ligériennes, Rapport du Programme PATRA (2010-2012), Coordination H. Davodeau. 65 p. (non publié).

Doyle M., Harbor J. M., Stanley E. H., 2003. Toward policies and decision-making for dam removal, Environmental Management, vol. 31, n 4, p. 453-465.

Fernandez S., Bouleau G., Treyer S., 2011. Reconsidérer la prospective de l'eau en Europe dans ses dimensions politiques. Développement durable et territoires, Vol. 2, $\mathrm{n}^{\circ}$ 3, décembre 2011 [http://developpementdurable.revues. org/9124].

Gatien A., Corbonnois J., Laurent F., 2009. Une analyse de paysage comme préalable à l'étude des systèmes agraires : application à la vallée du Loir, Norois, 4, n² 13, p. 89-101.

Germaine M.-A., 2009. De la caractérisation à la gestion des paysages ordinaires des vallées dans le Nord-Ouest de la France. Représentations, enjeux d'environnement et politiques publiques en Basse-Normandie. Caen, université de Caen Basse-Normandie, thèse de doctorat de géographie, 648 p.

Germaine M.-A., 2010. Trajectoires paysagères et poids des héritages dans les vallées normandes. Mappemonde $\mathrm{n}^{\circ} 99$, 3/2010 [http://mappemonde.mgm.fr/mum27/mois].

Germaine M.-A., 2011. Dépasser l'enjeu piscicole, vers la définition d'une gestion concertée du cours d'eau et de ses berges. L'exemple de la vallée de la Touques (Calvados), Géocarrefour, vol 86/3-4, p. 245-259.

Hydro Concept, 2003. Étude pré-opérationnelle à la restauration et à l'entretien du Loir, Rapport de pré-étude. 52 p. [www.sage-loir.fr].

Ouest-France, 2010. Visite rurale et campagnarde avec l'office du tourisme $d u$ Lude. Edition Pays de la Loire. Journal du 24 juillet [www.onest-france.fr].

Lescure S., Arnaud-fassetta G., Cordier S., 2011. Sur quelques modifications hydromorphologiques dans le val de Seine (Bassin Parisien, France) depuis 1830 : quelle part accorder aux facteurs hydrologiques et anthropiques? EchoGéo, 18, p 2-16.

Lespez L., Garnier E., Cador J.-M., Rocard D., 2005. Les aménagements hydrauliques et la dynamique des paysages des petits cours d'eau depuis le XVIII ${ }^{\mathrm{e}}$ siècle dans le NordOuest de la France : l'exemple du bassin versant de la Seulles (Calvados), La rivière aménagée entre héritages et modernité. Formes, techniques et mise en cuvre, Aestuaria, $\mathrm{n}^{\circ} 7$, p. 89-109.

Lespez L. (éd.). 2012. Paysages et gestion de l'eau. Sept millénaires d'histoire de vallées et de plaines littorales en basse Nor- mandie, Bibliothèque du Pole Rural n 3, MRSH-Presses universitaires de Caen, 336 p.

Olivier F., 2008. La roue du moulin n'arrête pas de tourner, Vivre à Vaas, n 49, p. 9-10.

Malavoi J.-R., 2003. Stratégie d'intervention de l'agence de l'eau sur les seuils en rivière, rapport d'étude, AREA - Loire-Bretagne, $135 \mathrm{p}$.

Micoud A., 2004. Des patrimoines aux territoires durables. Ethnologie et écologie dans les campagnes françaises, Ethnologie française, Vol. 34, p. 13-22.

Ministère de la Culture et de la Communication, 2006. Convention Vallée du Loir, Pays d'Art et d'Histoire, 18p [www. sarthe.gouv.fr/IMG/pdf/valoirconvention_cle18815f.pdf].

Montembault D., 2002. Les vallées face à l'appropriation urbaine. Des mutations de l'occupation du sol dans les vallées proches d'Angers aux nouveaux paysages. université d'Angers, thèse de doctorat de géographie, 2 tomes, 409 p.

Müller G., Koll K., 2004. River re-naturalization : historic and anthropogenic constraints, ecosystems and their interaction, In, Proceedings of the 5 th International Symposium on Ecohydraulics, Madrid, Spain, 12-17 Sep 2004, 5 p.

ONEMA, 2010. L'effacement total ou partiel d'obstacles transversaux, Rapport d'étude 90 p. [en ligne] [http://ftp.sandre.eaufrance.fr/ftp/incoming/AERMC/Metadonnees\%20AERMC_ Juillet\%202012/R111/46.pdf].

Pichot D., Margerie M., 2004. Approche pluridisciplinaire sur l'aménagement des petits cours d'eau par les sociétés médiévales dans l'ouest de la France ( $\mathrm{VII}^{\mathrm{e}}$-XVII ${ }^{\mathrm{e}}$ siècle), dans Burnouf J., Leveau P. (éd.) Flewves et marais, une histoire au croisement de la nature et de la culture. Sociétés préindustrielles et milieux fluviaux, lacustres et palustres : pratiques sociales et hydrosystèmes. CTHS, Paris, p. 111-125.

REPPAVAL, 2013. Représentation des paysages et de la nature dans les petites vallées de l'Ouest de la France face aux projets de restauration écologique [http://reppaval.hypotheses. org/ consulté le 15 mars 2013].

ONEMA 2008. Références nationales des Obstacles à l'Ecoulement (ROE). Base de données « obstacle à l'écoulement » [www.eau-loire-bretagne.fr].

Reynard E., 2005. Géomorphosites et paysages, Géomorphologie : relief, processus, environnement, $\mathrm{n}^{\circ} 3$, p. 181-188.

SAGE Loir, 2008. État des lieux du Loir, Novembre, 247 p. [www.sage-loir.fr].

Savatier E., 1989. Un industriel au XVIII ${ }^{\mathrm{e}}$ siècle, Revue historique et archéologique du Maine, Tome 46, Second semestre, $75 \mathrm{p}$.

Tснекро W., 2011. Étude des senils et moulins dans le bassin versant de l'Erve: Enjeux socio-économiques et environnementaux face à la Directive Cadre Européenne sur l'eau. Mémoire de Master 1, Université du Maine, 91 p.

Walter R. C., Merritts D. J., 2008. Natural streams and the legacy of water-powered Mills, Science, vol. 319, p. 299-304. 